\title{
Iterated integrals and higher order automorphic forms
}

\author{
Nikolaos Diamantis and Ramesh Sreekantan
}

\begin{abstract}
Higher order automorphic forms have recently been introduced to study important questions in number theory and mathematical physics. We investigate the connection between these functions and Chen's iterated integrals. Then using Chen's theory, we prove a structure theorem for automorphic forms of all orders. This allows us to define an analogue of a mixed Hodge structure on a space of higher order automorphic forms.
\end{abstract}

Mathematics Subject Classification (2000). 11F11, 14C30, 30F30, 32S35.

Keywords. Higher-order automorphic forms, Chen iterated integrals, mixed Hodge structures.

\section{Introduction}

In [4] the notion of Eisenstein series with modular symbols was introduced in order to study a new approach towards a conjecture of Szpiro. This series is not invariant under the action of the relevant group, but instead it satisfies a 4-term functional equation. Motivated by the applications of this Eisenstein series ([4], [5], [10], [11]), and the form of its functional equation which generalizes that of the classical automorphic forms, the first author and others began the general study of classes of functions satisfying equations of this type ([2]). Similar objects were defined and studied from a different viewpoint by Kleban and Zagier ([8]).

In this paper we complete the classification of a the space of automorphic forms of all orders and weights for a Fuchsian group of the first kind $\Gamma$ without elliptic elements along the lines of the classification of the space of second-order modular forms proved in [2]. It should be noted that the space classified here is larger than that studied in [2]. This was motivated by the desire to study certain automorphic forms that do not seem to belong to the smaller space.

For the classification we use Chen's theory of iterated integrals. Although it is possible that there exists an alternative proof of the classification that does not use iterated integrals, we wanted to highlight this connection with the important theory of iterated integrals. In this approach, higher order modular forms can be loosely viewed as antiderivatives of iterated integrals on the modular curve. (See [7], [9] for 
other applications of iterated integrals to modular forms which however do not deal with higher orders).

Based on this classification, we impose a Mixed-Hodge-type structure on the space of automorphic forms of all orders in the case of weight 0 . Because of the infinite dimensionality of the quotients of the "weight filtration", this structure is not a standard Mixed Hodge Structure.

However the structure described here reflects in a very natural way the algebraic structure of our space and it seems likely that certain subspaces of these automorphic forms could have a usual Mixed Hodge Structure.

Acknowledgment. The authors thank the referee for a very careful reading of the paper and for many useful suggestions.

\section{Higher order automorphic forms}

Let $\Gamma \subset \mathrm{PSL}_{2}(\mathbb{Z})$ be a Fuchsian group of the first kind with parabolic elements acting in the standard manner on the upper half-plane $\mathfrak{H}$. We use the set of generators of $\Gamma$ given by Fricke and Klein. Specifically, if $\Gamma \backslash \mathfrak{H}$ has genus $g, r$ elliptic fixed points and $m$ cusps, then there are $2 g$ hyperbolic elements $\gamma_{1}, \ldots, \gamma_{2 g}, m$ parabolic elements $\gamma_{2 g+1}, \ldots, \gamma_{2 g+m}$ and $r$ elliptic elements $\gamma_{2 g+m+1}, \ldots, \gamma_{2 g+m+r}$ generating $\Gamma$. Furthermore, these generators satisfy the $r+1$ relations:

$$
\left[\gamma_{1}, \gamma_{g+1}\right] \ldots\left[\gamma_{g}, \gamma_{2 g}\right] \gamma_{2 g+1} \ldots \gamma_{2 g+m} \gamma_{2 g+m+1} \ldots \gamma_{2 g+m+r}=1, \quad \gamma_{j}^{e_{j}}=1
$$

for $2 g+m+1 \leq j \leq 2 g+m+r$ and integers $e_{j} \geq 2$. Here $[a, b]$ denotes the commutator $a b a^{-1} b^{-1}$ of $a$ and $b$.

We set $Y(\Gamma)$ for the modular curve $\Gamma \backslash \mathfrak{H}$ and we consider the natural projection map $\pi: \mathfrak{H} \rightarrow Y(\Gamma)$. For a function $f$ on $\mathfrak{H}$ and an even integer $k$, set

$$
\left(\left.f\right|_{k} \gamma\right)(z):=(c z+d)^{-k} f(\gamma z)
$$

for $\gamma=\left(\begin{array}{ll}a & b \\ c & d\end{array}\right)$ in $\Gamma$. This defines an action of $\Gamma$ on the space of complex functions on $\mathfrak{H}$. We extend this action to $\mathbb{C}[\Gamma]$ by linearity.

A classical automorphic form of weight $k$ for $\Gamma$ is a smooth function $f$ of "at most polynomial growth at the cusps" such that

$$
\left.f\right|_{k}(\gamma-1)=0
$$

Let $J$ denote the augmentation ideal of the group ring which lies in the exact sequence

$$
0 \rightarrow J \rightarrow \mathbb{Z}[\Gamma] \stackrel{\operatorname{deg}}{\longrightarrow} \mathbb{Z} \rightarrow 0 .
$$


$J$ is generated by elements of the form $(\gamma-1)$ with $\gamma$ in $\Gamma$, so we can define a classical automorphic form as a function of "at most polynomial growth at the cusps" which is annihilated by $J$ via $\left.\right|_{k}$. We call holomorphic automorphic forms, modular.

With that in mind, we define an automorphic form $f$ of order $s$ to be a smooth function on $\mathfrak{H}$ such that

$$
\left.f\right|_{k} \delta=0 \quad \text { for all } \delta \in J^{s} .
$$

Let $M_{k}^{s}(\Gamma)$ denote the automorphic forms of weight $k$ and type $s$. From the definition, for a fixed $k$, we have

$$
M_{k}^{0}(\Gamma) \subseteq M_{k}^{1}(\Gamma) \subseteq M_{k}^{2}(\Gamma) \subseteq \cdots \subseteq M_{k}^{s}(\Gamma) .
$$

Classical automorphic forms are elements in $M_{k}^{1}(\Gamma)$ that satisfy certain growth conditions at the cusps. Of course, there are several variations of the definition of higher order modular forms. See [2], [3] for a related discussion.

The first step towards the classification of automorphic forms of order $s$ is

Proposition 2.1. Let

$$
\psi: M_{k}^{s+1} \rightarrow \bigoplus_{i=1}^{(2 g+m-1)^{s}} M_{k}^{1}
$$

be defined by

$$
\psi(f)=\left(\left.f\right|_{k}\left(\gamma_{i_{1}}-1\right) \ldots\left(\gamma_{i_{s}}-1\right)\right)_{1 \leq i_{1}, \ldots, i_{s} \leq 2 g+m-1} .
$$

Then

$$
0 \rightarrow M_{k}^{s} \hookrightarrow M_{k}^{s+1} \stackrel{\psi}{\rightarrow} \bigoplus_{i=1}^{(2 g+m-1)^{s}} M_{k}^{1}
$$

is an exact sequence.

Proof. To prove that $\operatorname{ker}(\psi) \subset M_{k}^{s}$, we first observe that, if $f \in M_{k}^{s+1}$, then for each $\delta_{1} \in J^{m}, \delta_{2} \in J^{s-m-1}$, we have

$$
\left.f\right|_{k} \delta_{1}\left(\gamma_{1} \gamma_{2}-1\right) \delta_{2}=\left.f\right|_{k} \delta_{1}\left(\gamma_{1}-1\right) \delta_{2}+\left.f\right|_{k} \delta_{1}\left(\gamma_{2}-1\right) \delta_{2} .
$$

This follows from the observation that

$$
\gamma_{1} \gamma_{2}-1=\left(\gamma_{1}-1\right)\left(\gamma_{2}-1\right)+\left(\gamma_{1}-1\right)+\left(\gamma_{2}-1\right) .
$$

Now let $f$ be in $\operatorname{ker}(\psi)$. Using (1) we observe that to prove $f$ is in $M_{k}^{s}(\Gamma)$, it suffices to verify that

$$
\left.f\right|_{k}\left(g_{1}-1\right) \ldots\left(g_{s}-1\right)=0
$$

for each $s$-tuple of generators $g_{i}$ of $\Gamma$. 
From the definition of $\psi,\left.f\right|_{k}\left(g_{1}-1\right) \ldots\left(g_{s}-1\right)=0$ for all the non-elliptic generators $g_{i} \in\left\{\gamma_{1}, \ldots, \gamma_{2 g+m-1}\right\}$ of $\Gamma$.

Further, let $\gamma \in \Gamma$ be one of the elliptic generators with $\gamma^{e}=1$. As $\delta_{1}\left(\gamma^{l}-1\right)$ is in $J^{m+1}$, for every $\delta_{1} \in J^{m}$, we have

$$
\left.f\right|_{k} \delta_{1} \gamma^{l}(\gamma-1) \delta_{2}=\left.f\right|_{k} \delta_{1}(\gamma-1) \delta_{2}
$$

for $\delta_{1} \in J^{m}, \delta_{2} \in J^{s-m-1}, l=0, \ldots e-1$. Therefore,

$$
\left.e f\right|_{k} \delta_{1}(\gamma-1) \delta_{2}=\left.f\right|_{k} \delta_{1}\left(\sum_{l=0}^{e-1} \gamma^{l}\right)(\gamma-1) \delta_{2}=\left.f\right|_{k} \delta_{1}\left(\gamma^{e}-1\right) \delta_{2}=0
$$

so

$$
\left.f\right|_{k} \delta_{1}(\gamma-1) \delta_{2}=0 \quad \text { for all } \delta_{1} \in J^{m}, \delta_{2} \in J^{s-m-1} .
$$

Finally, using the relation between the generators, we can write

$$
\gamma_{2 g+m}=\left(\left[\gamma_{1}, \gamma_{g+1}\right] \ldots\left[\gamma_{g}, \gamma_{2 g}\right] \gamma_{2 g+1} \ldots \gamma_{2 g+m-1}\right)^{-1}\left(\gamma_{2 g+m+1} \ldots \gamma_{2 g+m+r}\right)^{-1}
$$

and, by (2), $\gamma_{2 g+m+1}-1$ can be expressed in terms of the other generators. This implies that $\left.f\right|_{k}\left(g_{1}-1\right) \ldots\left(g_{s}-1\right)=0$ for all $g_{i}$ 's in the set of generators of $\Gamma$ and the middle term of the sequence is exact.

The surjectivity of $\psi$ will be studied in Section 4 . To this end, we will need to define Chen's iterated integrals and to review their basic properties.

\section{Iterated integrals}

Let $X$ be a smooth manifold. Let $P(X)$ denote the space of paths on $X$, namely piecewise smooth

$$
\gamma:[0,1] \rightarrow X
$$

A function $\phi: P(X) \rightarrow \mathbb{C}$ is said to be a homotopy functional if $\phi$ depends only on the homotopy class of $\gamma$ relative to its endpoints, that is, it defines a function on $\Gamma=\pi_{1}\left(X, x_{0}\right)$, where $x_{0}$ is a fixed point of $X$. Equivalently, it induces an element of $\operatorname{Hom}(\mathbb{Z}[\Gamma], \mathbb{C})$.

Let $w$ be a smooth 1 -form on $X$. The map

$$
\gamma \rightarrow \int_{\gamma} w=\int_{0}^{1} f(t) d t
$$

where $\gamma^{*}(w)=f(t) d t$, defines a function on $P(X)$. This defines an element of $\operatorname{Hom}(\mathbb{Z}[\Gamma], \mathbb{C})$ if and only if $w$ is closed. Hence this only detects elements of $\Gamma$ 
visible in the homology of $X$ - it vanishes on $J^{2}(J$ denotes the augmentation ideal of $\mathbb{Z}\left[\pi_{1}\left(X, x_{0}\right)\right]$ here $)$.

The iterated integrals studied by Chen (e.g. [1]) detect more elements of the group ring. Specifically, suppose that $w_{1}, w_{2}, \ldots, w_{r} \in E^{1}(X)$, where $E^{1}(X)$ denotes the space of smooth 1 -forms on $X$. We will write

$$
w_{1} \ldots w_{s}=w_{1} \otimes \cdots \otimes w_{s} \in \bigotimes^{s} E^{1}(X)
$$

and call it a "product" of the $w_{i}$ 's. We set $w_{1} \ldots w_{s}=1$, when $s=0$.

If $\gamma$ is a path on $X$, we set

$$
\int_{\gamma} w_{1} w_{2} \ldots w_{r}=\int \ldots \int_{0 \leq t_{1} \leq t_{2} \cdots \leq t_{r} \leq 1} f_{1}\left(t_{1}\right) f_{2}\left(t_{2}\right) \ldots f_{r}\left(t_{r}\right) d t_{1} d t_{2} \ldots d t_{r},
$$

where $\gamma^{*}\left(w_{i}\right)=f_{i}(t) d t$. This defines a function on the space of paths of $X$ which will be denoted by $\int w_{1} \ldots w_{r}$ and is called a iterated line integral of length $r$. A linear combination of such functions is called an iterated integral and its length is the length of the longest line integral. However, it is not necessarily a homotopy functional.

Let $B_{S}(X)$ denote the space of iterated integrals of length $\leq s$. If $\mathcal{I}$ is in $B_{S}(X)$ and $\alpha \in P(X)$ we denote the evaluation map by $\langle\mathcal{I}, \alpha\rangle$. We extend it to all 1 -chains by linearity.

The next theorem states that in some cases an iterated integral can be modified to be a homotopy functional.

Theorem 3.1 (Chen [1], Section 3). Let $X$ be a connected, smooth manifold with $H^{2}(X)=0$ and let $w_{1}, \ldots w_{s}$ be closed 1 -forms on $X$. Then there is an $\mathcal{I} \in B_{S}(X)$ which is a homotopy functional and a $\mathcal{K} \in B_{s-1}(X)$ satisfying

$$
\langle\mathcal{I}, \alpha\rangle=\int_{\alpha} w_{1} \ldots w_{s}+\langle\mathcal{K}, \alpha\rangle,
$$

for each path $\alpha \in P(X)$.

Proof. An example of an $\mathscr{I}$ satisfying these conditions can be constructed using an extended defining system for a Massey product of $w_{1}, \ldots, w_{s}$ : Fix smooth 1-forms $w_{12}, w_{23}, \ldots, w_{123}, \ldots$ such that

$$
\begin{gathered}
w_{1} \wedge w_{2}+d w_{12}=0, \ldots, w_{s-1} \wedge w_{s}+d w_{(s-1) s}=0, \ldots \\
w_{1} \wedge w_{23}+w_{12} \wedge w_{3}+d w_{123}=0, \ldots \\
w_{1} \wedge w_{2 \ldots s}+w_{12} \wedge w_{3 \ldots s}+\cdots+d w_{1 \ldots s}=0 .
\end{gathered}
$$


We then set

$$
\tau=\int u
$$

where

$$
\begin{array}{r}
u:=w_{1} w_{2} \ldots w_{s}+w_{12} w_{3} \ldots w_{s}+w_{1} w_{23} \ldots w_{s}+\ldots \\
+w_{123} w_{4} \ldots w_{s}+w_{1} w_{234} \ldots w_{s}+\cdots+w_{1 \ldots s} .
\end{array}
$$

Note that $\int u-\int w_{1} w_{2} \ldots w_{s}$ is of length $<s$. The proof of the independence of path can be found in [1], page 366 .

If $w$ is a 1 -form and $\alpha, \beta$ are two loops based at $x_{0}$, then it is easy to see

$$
\left\langle\int w,(\alpha-1)(\beta-1)\right\rangle=0 \text {. }
$$

We will need the following lemmas. The first lemma generalizes the above comment.

Lemma 3.2 ([6], Lemma 2.10, Proposition 2.13). Let $w_{1}, \ldots w_{r}$ be smooth 1-forms on $X$ and let $\alpha=\prod_{i=1}^{s}\left(\alpha_{i}-1\right)$, where $\alpha_{i}$ are loops based at $x_{0}$. Then

$$
\left\langle\int w_{1} \ldots w_{r}, \alpha\right\rangle= \begin{cases}0 & \text { if } r<s, \\ \prod_{i=1}^{s} \int_{\alpha_{i}} w_{i} & \text { if } r=s .\end{cases}
$$

The second lemma describes what happens under composition of paths.

Lemma 3.3 ([6], Proposition 2.9). Let $w_{1}, \ldots w_{s}$ be smooth 1-forms on $X$ and let $\alpha, \beta$ be paths such that $\alpha(1)=\beta(0)$. Then

$$
\begin{aligned}
\left\langle\int w_{1} \ldots w_{s}, \alpha \beta\right\rangle=\langle & \left.\int w_{1} \ldots w_{s}, \alpha\right\rangle+\left\langle\int w_{1} \ldots w_{s}, \beta\right\rangle \\
& +\sum_{j=1}^{s-1}\left\langle\int w_{1} \ldots w_{j}, \alpha\right\rangle\left\langle\int w_{j+1} \ldots w_{s}, \beta\right\rangle .
\end{aligned}
$$

An application of Lemma 3.3 and Theorem 3.1 is the following.

Lemma 3.4. Let $X$ be a connected, smooth manifold with $H^{2}(X)=0$ and let $w_{1}, \ldots w_{s}$ be closed 1 -forms on $X$. If $\left\{w_{12}, \ldots, w_{(s-1) s}, \ldots, w_{1 \ldots s}\right\}$ is an extended defining system for a Massey product of $w_{1}, \ldots w_{s}$, we set

$$
\begin{aligned}
& u_{j}=w_{1} \ldots w_{j}+w_{12} w_{3} \ldots w_{j}+w_{1} w_{23} \ldots w_{j}+\cdots+w_{1 \ldots j}, \quad j=1, \ldots s \\
& u^{j}=w_{j+1} \ldots w_{s}+w_{(j+1)(j+2)} w_{j+3} \ldots w_{s} \\
& +w_{j+1} w_{(j+2)(j+3)} \ldots w_{s}+\cdots+w_{j+1 \ldots s} \quad j=1, \ldots s-1 .
\end{aligned}
$$


We also set $u:=u_{s}$. Then for each pair of paths $\alpha$ and $\beta$ on $X$ with $\alpha(1)=\beta(0)$, we have

$$
\int_{\alpha \beta} u=\int_{\alpha} u+\int_{\beta} u+\sum_{j=1}^{s-1} \int_{\alpha} u_{j} \int_{\beta} u^{j} .
$$

Proof. From the construction of $u$, all combinations of $1,2, \ldots, s$ appear in $u$ (in this order) as indices of "products" of $w$ 's. Applying Lemma 3.3, we can decompose the integral of each individual "product" as a sum of products of iterated integrals on $\alpha$ and on $\beta$. Thus,

$$
\int_{\alpha \beta} u=\sum_{j=0}^{s}\left(\sum_{v_{j}}\left(\sum_{v^{j}} \int_{\alpha} v_{j} \int_{\beta} v^{j}\right)\right)
$$

where $\sum_{v_{j}}\left(\operatorname{resp} . \sum_{v^{j}}\right)$ ranges over all "products" $v_{j}\left(\right.$ resp. $\left.v^{j}\right)$ with index sequences formed by the integers $1, \ldots, j$ (resp. $j+1, \ldots s$ ). (Here we have set $\int v_{0}=$ $\int v^{s+1} \equiv 1$.) For example $\sum_{v_{3}} \int_{\alpha} v_{3}=\int_{\alpha}\left(w_{1} w_{2} w_{3}+w_{1} w_{23}+w_{12} w_{3}+w_{123}\right)$.

From the defining equations of $u_{j}$ and $u^{j}$,

$$
\sum_{v_{j}} \int_{\alpha} v_{j}=\int_{\alpha} u_{j} \text { and } \sum_{v^{j}} \int_{\beta} v^{j}=\int_{\beta} u^{j} .
$$

This proves the identity.

\section{The classification of higher order automorphic forms}

We now restrict ourselves to the case when $X=Y(\Gamma)$, where $\Gamma \subset \mathrm{PSL}_{2}(\mathbb{Z})$ is a Fuchsian group of the first kind with parabolic elements, as in Section 2. In addition, we assume that $\Gamma$ has no elliptic elements and hence $\pi_{1}(X)=\Gamma$. In this section, we complete the classification of the vector space of automorphic forms of order $s$ and weight $k$ for such groups. We first work in the case of weight 0 proving what amounts to a variation of the general statement we will eventually prove.

Let $\tilde{M}_{0}^{s+1}=\tilde{M}_{0}^{s+1}(\Gamma)$ denote the space of $f \in M_{0}^{s+1}=M_{0}^{s+1}(\Gamma)$ such that $\left.f\right|_{0} \delta \in \mathbb{C}$ for every $\delta$ in $J^{S}$.

Proposition 4.1. The sequence

$$
0 \rightarrow M_{0}^{s} \hookrightarrow \tilde{M}_{0}^{s+1} \stackrel{\psi}{\rightarrow} \bigoplus_{i=1}^{(2 g+m-1)^{s}} \mathbb{C} \rightarrow 0
$$

with $\psi$ defined as in Proposition 2.1 is exact. 
Proof. The exactness of the middle term follows from Proposition 2.1 because $\tilde{M}_{0}^{s+1} \subset M_{0}^{s+1}$. From that, we observe that to prove surjectivity, it suffices to construct $(2 g+m-1)^{s}$ linearly independent elements of $\tilde{M}_{0}^{s+1} / M_{0}^{s}$.

Let $\left\{f_{1}, \ldots, f_{g}, g_{1}, \ldots, g_{m-1}\right\}$ be a basis of the space of holomorphic modular forms of weight 2 for $\Gamma$ with $f_{i}$ cuspidal and $g_{i}$ non-cuspidal. Let $\left\{w_{i}\right\}_{i=1}^{g}$ be the differentials on $Y(\Gamma)$ corresponding to $\left\{f_{i}(z) d z\right\}_{i=1}^{g},\left\{w_{i}\right\}_{i=g+1}^{2 g}$ the differentials corresponding to $\left\{\overline{f_{i-g}(z)} d \bar{z}\right\}_{i=g+1}^{2 g}$ (where the bar stands for complex conjugation) and $\left\{w_{2 g+i}\right\}_{i=1}^{m-1}$ the differentials corresponding to $\left\{g_{i}(z) d z\right\}_{i=1}^{m-1}$.

Then, all $w_{i}$,'s are closed, as are $w_{i} \wedge w_{j}(i, j=1, \ldots, 2 g+m-1)$. Furthermore, since $Y(\Gamma)$ is non-compact, from the Gysin exact sequence, we have

$$
H^{2}(Y(\Gamma), \mathbb{C})=0 .
$$

Hence we can apply Theorem 3.1 to any selection of $s$ forms from $\left\{w_{i} ; i=1, \ldots\right.$, $2 g+m-1\}$. Let $I=\left(i_{1}, i_{2}, \ldots, i_{s}\right)$ be any indexing vector with elements in $\{1, \ldots, 2 g+m-1\}$ and let $\mathcal{I}_{I}, \mathcal{K}_{I}$ and $u_{I}$ be induced by $\left\{w_{i_{j}}\right\}_{j=1}^{s}$ as in the proof of Theorem 3.1. Let $x_{0}$ be a point in the upper half-plane lying over a point, which we will also denote by $x_{0}$ on the curve. If we let $\left\{x_{0}, b\right\}$ denote the image under $\pi$ of the line path from $x_{0}$ to $b$ in $\mathfrak{H}$, then the function

$$
F_{\chi_{I}}(z):=F_{I}(z):=\left\langle\mathcal{I}_{I},\left\{x_{0}, z\right\}\right\rangle, \quad z \in \mathfrak{H}
$$

is well defined and independent of the path from $x_{0}$ to $z$, since $\pi$ maps homotopic paths to homotopic paths.

We will now show the $F_{I}$ 's are in $\tilde{M}_{0}^{s+1}(\Gamma)$ for each $I$. In Lemma 4.3 we will further show that they are linearly independent modulo $M_{0}^{s}(\Gamma)$. As there are $(2 g+m-1)^{s}$ of them, these two facts will suffice to prove Proposition 4.1.

We first use Lemma 3.4 to compute $\left.F_{I}\right|_{0} \delta,\left(\delta \in J^{s}\right)$ and show that it is a constant. We use the notation $\{a, b\}$ for the path $\left\{a, x_{0}\right\}$ followed by $\left\{x_{0}, b\right\}$.

Lemma 4.2. Let $I=\left(i_{1}, \ldots, i_{s}\right)$ be an indexing vector with elements in $\{1, \ldots$, $2 g+m-1\}$ and set $F_{I}(z)=\left\langle\mathcal{I}_{I},\left\{x_{0}, z\right\}\right\rangle$. Then, for any $\delta=\prod_{k=1}^{s}\left(\gamma_{k}-1\right) \in J^{s}$,

$$
\left(\left.F_{I}\right|_{0} \delta\right)(z)=\prod_{k=1}^{s} \int_{\left\{z, \gamma_{k} z\right\}} w_{i_{k}} .
$$

In particular, since the $w_{i}$ correspond to classical modular forms of weight 2 and their conjugates, this expression is independent of $z$ and so $F_{I}(z) \in \tilde{M}_{0}^{s+1}(\Gamma)$.

Proof. For every $\gamma \in \Gamma$,

$$
\left.F_{I}\right|_{0}(\gamma-1)(z)=\left\langle\mathcal{I}_{I},\left\{x_{0}, \gamma z\right\}\right\rangle-\left\langle\mathcal{I}_{I},\left\{x_{0}, z\right\}\right\rangle .
$$


Combining this with Lemma 3.4 with $\alpha=\left\{x_{0}, z\right\}$ and $\beta=\{z, \gamma z\}$ gives

$$
\left.F_{I}\right|_{0}(\gamma-1)(z)=\left\langle\mathcal{I}_{I},\{z, \gamma z\}\right\rangle+\sum_{j=1}^{s-1} \int_{\left\{x_{0}, z\right\}} u_{j} \int_{\{z, \gamma z\}} u^{j}
$$

Observe that, if $\gamma_{1}, \ldots, \gamma_{s}$ are in $\Gamma$, we have the following expressing generalizing (2):

$$
\prod_{k=1}^{s}\left(\gamma_{k}-1\right)=\left(\gamma_{1} \ldots \gamma_{s}-1\right)+\cdots+(-1)^{s-1}\left(\gamma_{1}-1\right) \cdots+(-1)^{s-1}\left(\gamma_{s}-1\right) \text {. }
$$

Combining this with (6), we have

$$
\begin{aligned}
& \left.F_{I}\right|_{0}\left(\gamma_{1}-1\right) \ldots\left(\gamma_{s}-1\right)(z) \\
& =\left.F_{I}\right|_{0}\left(\left(\gamma_{1} \ldots \gamma_{s}-1\right)+\cdots+(-1)^{s-1}\left(\gamma_{s}-1\right)\right)(z) \\
& =\left\langle\mathcal{I}_{I},\left\{z, \gamma_{1} \ldots \gamma_{s} z\right\}\right\rangle+\cdots+(-1)^{s-1}\left\langle\mathcal{I}_{I},\left\{z, \gamma_{s} z\right\}\right\rangle \\
& \quad+\sum_{j=1}^{s-1} \int_{\left\{x_{0}, z\right\}} u_{j}\left(\int_{\left\{z, \gamma_{1} \ldots \gamma_{s} z\right\}} u^{j}+\cdots+(-1)^{s-1} \int_{\left\{z, \gamma_{s} z\right\}} u^{j}\right) .
\end{aligned}
$$

Next we observe that, in $\mathfrak{H}$, the path from $\gamma z$ to $\gamma \delta z$ passing through $x_{0}$ is homotopic to $\gamma(z, \delta z)$, where $(z, \delta z)$ is the path from $z$ to $\delta z$ passing through $x_{0}$. Since the image of $\gamma(z, \delta z)$ under $\pi$ is $\{z, \delta z\}$, the loops $\{\gamma z, \gamma \delta z\}$ and $\{z, \delta z\}$ are homotopic in $X$ and hence $\{z, \gamma \delta z\}$ is homotopic to $\{z, \gamma z\}\{z, \delta z\}$. On the other hand, by the proof of Theorem 3.1, each $\int u^{j}$ is homotopy invariant. Therefore, by induction, for all $\gamma_{1}, \ldots \gamma_{s} \in \Gamma$, we have

$$
\begin{aligned}
& \left.F_{I}\right|_{0}\left(\gamma_{1}-1\right) \ldots\left(\gamma_{s}-1\right)(z) \\
& =\left\langle\mathcal{I}_{I},\left(\left\{z, \gamma_{1} z\right\}-1\right) \ldots\left(\left\{z, \gamma_{s} z\right\}-1\right)\right\rangle \\
& \quad+\left(\sum_{j=1}^{s-1}\left\langle\int u_{j},\left\{x_{0}, z\right\}\right\rangle\left\langle\int u^{j},\left(\left\{z, \gamma_{1} z\right\}-1\right) \ldots\left(\left\{z, \gamma_{s} z\right\}-1\right)\right\rangle\right) .
\end{aligned}
$$

Since the iterated integrals in the sum within the parenthesis are of length $<s$ and since $\mathcal{I}_{I} \equiv \prod_{k=1}^{s} \int w_{i_{k}}$ up to an iterated integral of length $<s$, by Lemma (3.2) we deduce

$$
\left.F_{I}\right|_{0}\left(\gamma_{1}-1\right) \ldots\left(\gamma_{s}-1\right)(z)=\int_{\left\{z, \gamma_{1} z\right\}} w_{i_{1}} \ldots \int_{\left\{z, \gamma_{s} z\right\}} w_{i_{s}} .
$$

To complete the proof of Proposition 4.1 we show that the images of $F_{I}$ under the natural projection

are linearly independent.

$$
\tilde{M}_{0}^{s+1} \rightarrow \tilde{M}_{0}^{s+1} / M_{0}^{s}
$$


Lemma 4.3. Suppose there exist complex numbers $k_{I}$ satisfying

$$
\sum_{I} k_{I} F_{I} \in M_{0}^{s}(\Gamma)
$$

where I runs through the $(2 g+m-1)^{s}$ possible s-tuples of $\{1, \ldots, 2 g+m-1\}$. Then $k_{I}=0$ for all $I$.

Proof. We proceed by induction. Suppose $s=1$ and that there are complex numbers $k_{r}$ such that

$$
\sum_{r=1}^{2 g+m-1} k_{r} F_{r} \in M_{0}^{1}(\Gamma) .
$$

Then, from Lemma 4.2, for any $\gamma \in \Gamma$ we have

$$
\left.\sum_{r=1}^{2 g+m-1} k_{r} F_{r}\right|_{0}(\gamma-1)(z)=\sum_{r=1}^{2 g+m-1} k_{r} \int_{\{z, \gamma z\}} w_{r}=0
$$

where $w_{r}$ are the (holomorphic or anti-holomorphic) differential forms corresponding to the basis of the space of weight 2 modular forms for $\Gamma$ we fixed at the beginning of the proof of Proposition 4.1. From the injectivity of the classical Eichler-Shimura isomorphism, we have $k_{r}=0$ for all $r$.

Proceeding by induction, suppose there are complex numbers $k_{I}$ satisfying

$$
\sum_{I} k_{I} F_{I} \in M_{0}^{s}
$$

where $I$ runs through the $(2 g+m-1)^{s}$ possible $s$-tuples $\left(i_{1}, \ldots, i_{s}\right)$ of the set $\{1, \ldots, 2 g+m-1\}$. Then

$$
\left.\sum_{I} k_{I} F_{I}\right|_{0}\left(\gamma_{1}-1\right) \ldots\left(\gamma_{s}-1\right)=0
$$

for any $\gamma_{1}, \ldots, \gamma_{s}$ in $\Gamma$.

Applying Lemma 4.2, this is equivalent to

$$
\sum_{I} k_{I} \int_{\left\{z, \gamma_{1} z\right\}} w_{i_{1}} \ldots \int_{\left\{z, \gamma_{s} z\right\}} w_{i_{s}}=0
$$

or

$$
\begin{aligned}
& \sum_{i_{s}=1}^{2 g+m-1}\left(\sum_{i_{s-1}=1}^{2 g+m-1} \cdots \sum_{i_{1}=1}^{2 g+m-1} k_{I} \int_{\left\{z, \gamma_{1} z\right\}} w_{i_{1}} \cdots \int_{\left\{z, \gamma_{s-1} z\right\}} w_{i_{s-1}}\right) \int_{\left\{z, \gamma_{s} z\right\}} w_{i_{s}} \\
& \quad=\sum_{i_{s}=1}^{2 g+m-1} A_{i_{s}} \int_{\left\{z, \gamma_{s} z\right\}} w_{i_{s}}=0 .
\end{aligned}
$$


From the Eichler-Shimura isomorphism once again, we have that $A_{i_{s}}=0$ for all $i_{s}$. For a fixed $i_{s}, A_{i_{s}}$ is

$$
\sum_{I^{\prime}} k_{I^{\prime} \cup\left\{i_{s}\right\}} \int_{\left\{z, \gamma_{1} z\right\}} w_{i_{1}} \ldots \int_{\left\{z, \gamma_{s-1} z\right\}} w_{i_{s-1}}
$$

where $I^{\prime}$ runs through all possible $(s-1)$-tuples. By induction,

$$
k_{I^{\prime} \cup\left\{i_{s}\right\}}=0 .
$$

As this is true for all $i_{s}, k_{I}=0$ for all $I$ and the $F_{I}$ are linearly independent modulo $M_{0}^{s}(\Gamma)$.

This completes the proof of Proposition 4.1.

The following classification theorem is an application of Proposition 4.1.

Theorem 4.4. Let

$$
\psi: M_{k}^{s+1} \rightarrow \bigoplus_{i=1}^{(2 g+m-1)^{s}} M_{k}^{1}
$$

be defined by

$$
\psi(f)=\left(\left.f\right|_{k}\left(\gamma_{i_{1}}-1\right) \ldots\left(\gamma_{i_{s}}-1\right)\right)_{1 \leq i_{1}, \ldots, i_{s} \leq 2 g+m-1}
$$

Then,

$$
0 \rightarrow M_{k}^{s} \hookrightarrow M_{k}^{s+1} \stackrel{\psi}{\rightarrow} \bigoplus_{i=1}^{(2 g+m-1)^{s}} M_{k}^{1} \rightarrow 0
$$

is an exact sequence.

Proof. In view of Proposition 2.1, the only part that needs to be proved is the surjectivity of $\psi$. Let

$$
\left(f_{I}\right)_{I} \in \bigoplus_{i=1}^{(2 g+m-1)^{s}} M_{k}^{1}
$$

with $I$ ranging through the $(2 g+m-1)^{s}$ possible $s$-tuples of $\{1, \ldots, 2 g+m-1\}$. We will show that there is a $F \in M_{k}^{s+1}$ such that $\psi(F)=\left(f_{I}\right)_{I}$ or equivalently,

$$
\left.F\right|_{k}\left(\gamma_{i_{1}}-1\right) \ldots\left(\gamma_{i_{s}}-1\right)=f_{I}
$$

for all $s$-tuples $I=\left\{i_{i}, \ldots, i_{s}\right\}$. 
From the surjectivity part of Theorem 4.1, for each $s$-tuple of integers $L=$ $\left(l_{1}, \ldots l_{s}\right)$ with $l_{j} \in\{1, \ldots, 2 g+m-1\}$, there is a $\Lambda_{L} \in \tilde{M}_{0}^{s+1}$ such that

$$
\left.\Lambda_{L}\right|_{0}\left(\gamma_{i_{1}}-1\right) \ldots\left(\gamma_{i_{s}}-1\right)=\delta_{I}^{L}
$$

for any $s$-tuple $I$, where $\delta_{I}^{L}$ is the Kronecker delta function of the $s$-tuple, namely $\prod_{k} \delta_{i_{k}}^{l_{k}}$

An easy computation then shows that

$$
F=\sum_{L} f_{L} \Lambda_{L}
$$

is in $M_{k}^{s+1}$ and satisfies the desired equality.

We shall finally use the last two propositions to show that the direct sum of all $M_{0}^{s}$ can be endowed with a structure which in some respects is very similar to a Mixed Hodge Structure.

Theorem 4.5. Set

$$
V=\bigoplus_{s=1}^{\infty} M_{0}^{s}
$$

There exists

- a increasing filtration $W_{\bullet}$ on $V$ and

- a decreasing filtration $F^{\bullet}$ on $V$

such that, for each $m \in \mathbb{N}$, the filtration on $G r_{m}\left(W^{\bullet}\right)=W_{m} / W_{m-1}$ defined by

$$
F^{p}\left(G r_{m}\left(W^{\bullet}\right)\right)=\left(W_{m} \cap F^{p}\right) /\left(W_{m-1} \cap F^{p}\right)
$$

satisfies

$$
F^{p}\left(G r_{m}\left(W^{\bullet}\right)\right) \oplus \bar{F}^{m-p+1}\left(G r_{m}\left(W^{\bullet}\right)\right) \cong G r_{m}\left(W^{\bullet}\right)
$$

Proof. It suffices to construct two filtrations $W_{\bullet}$ and $F^{\bullet}$ satisfying the following property: If $w \in W_{m}$ and $p, q$ are integers such that $p+q=m+1$, then there exist $w_{1} \in F^{p} \cap W_{m}, w_{2} \in F^{q} \cap W_{m}$ and $w_{0} \in W_{m-1}$ such that $w=w_{1}+\bar{w}_{2}+w_{0}$. Moreover, this decomposition is unique modulo $W_{m-1}$.

We define $W_{\bullet}$ setting $W_{m}=M_{0}^{m+1}$.

To define $F^{\bullet}$ we first consider the set $\mathfrak{F}$ of all functions that are induced by modular forms of weight 2 and their conjugates as in (5). Specifically, $\mathfrak{F}$ contains all functions $f: \mathfrak{H} \rightarrow \mathbb{C}$ with the property that

$$
f(z)=\left\langle\mathcal{L},\left\{x_{0}, z\right\}\right\rangle, \quad \text { for all } z \in \mathfrak{H},
$$


for a homotopy functional $\mathcal{L} \in B_{S}(X)$ such that

$$
\tau \equiv \int w_{1} \ldots w_{s} \bmod B_{s-1}(X)
$$

for some $w_{i}$ corresponding to weight 2 modular forms and their conjugates. We do not require that the modular forms inducing $f$ belong to the basis fixed in the proof of Proposition 4.1.

We next consider the set $\mathfrak{F}^{p}$ of all $F \in \mathfrak{F}$ induced by forms $w_{i}$ among which at least $p$ are holomorphic. $F^{p}$ is then defined as the space generated by products of the form $f \cdot F$, with $f$ in $M_{0}^{1}$ and $F \in \mathfrak{F}^{p}$, i.e.

$$
F^{p}:=\left\langle f \cdot F ; f \in M_{0}^{1}, F \in \mathfrak{F}^{p}\right\rangle .
$$

Now, Theorem 4.4 implies that each $F \in W_{m}=M_{0}^{m+1}$ can be expressed as a sum of functions of the form $f_{L} \cdot \Lambda_{L}$, where $L$ is an indexing vector of length $m$ and $f_{L} \in$ $M_{0}^{1}$. By the construction of $\Lambda_{L}$, each of them can be expressed, uniquely modulo a function in $M_{0}^{m}$, as a linear combination of the $F_{I}$ 's constructed in Proposition 4.1. Here $I$ is an indexing set of length $m$. Therefore, $F$ can be written in the form

$$
F=\sum_{I} f_{I} F_{I}+F_{0}
$$

for some $F_{0} \in W_{m-1}=M_{0}^{m}$ and $f_{I} \in M_{0}^{1}$.

Let $p, q$ be positive integers such that $p+q=m+1$ and let $F_{I}$ be one of the functions in the right-hand side of (8). If the set of 1-forms inducing $F_{I}$ contains less than $p$ holomorphic forms, then it contains at least $q=m-p+1$ anti-holomorphic ones. In addition, by the construction of $\mathcal{I}_{I}$ 's and $F_{I}$ 's, $\bar{F}_{I} \in \mathfrak{F}$. Hence $\bar{F}_{I}$ is induced by at least $q$ holomorphic forms and $\overline{f_{I} F_{I}} \in F^{q}$. This completes the proof.

As mentioned above, this structure is not quite a MHS mainly as the $G r_{m}\left(W^{\bullet}\right)$ 's are not finite dimensional. It is also not clear that the definition is functorial. However, it appears that there is an interesting subspace which has a genuine mixed Hodge structure. We will discuss this in a future paper.

\section{References}

[1] K.-T. Chen, Algebras of iterated path integrals and fundamental groups. Trans. Amer. Math. Soc. 156 (1971), 359-379. Zbl 0217.47705 MR 0275312

[2] G. Chinta, N. Diamantis, C. O'Sullivan, Second order modular forms. Acta Arith. 103 (2002), 209-223. Zbl 1020.11025 MR 1905087

[3] N. Diamantis, M. Knopp, G. Mason, C. O'Sullivan, L-functions of second order modular forms. The Ramanujan Journal, to appear. 
[4] D. Goldfeld, The distribution of modular symbols. In Number theory in progress (Zakopane-Kościelisko, 1997), Vol. 2, Elementary and analytic number theory, Walter de Gruyter, Berlin 1999, 849-865. Zbl 0948.11022 MR 1689548

[5] D. Goldfeld, Zeta functions formed with modular symbols. In Automorphic forms, automorphic representations, and arithmetic (Fort Worth, TX, 1996), Proc. Sympos. Pure Math. 66, Part 1, Amer. Math. Soc., Providence, RI, 1999, 111-121. Zbl 0934.11026 MR 1703748

[6] R. Hain, The geometry of the mixed Hodge structure on the fundamental group. In Algebraic geometry (Brunswick, Maine, 1985), Proc. Sympos. Pure Math. 46, Part 2, Amer. Math. Soc., Providence, RI, 1987, 247-282. Zbl 0654.14006 MR 0927984

[7] B. Harris, Iterated integrals and cycles on algebraic manifolds. Nankai Tracts in Mathematics 7, World Scientific Publishing Co., Inc., River Edge, NJ, 2004. Zbl 1063.14010 MR 2063961

[8] P. Kleban, D. Zagier, Crossing probabilities and modular forms. J. Statist. Phys. 113 (2003), 431-454. Zbl 02012872 MR 2013692

[9] Y. Manin, Iterated integrals of modular forms and noncommutative modular symbols. arXiv:math.NT/0502576.

[10] Y. Petridis, M. Risager, Modular symbols have a normal distribution. Geom. Funct. Anal. 14 (2004), 1013-1043. Zbl 02138258 MR 2105951

[11] M. Risager, On the distribution of modular symbols for compact surfaces. Internat. Math. Res. Notices 41 (2004), 2125-2146. Zbl 02150556 MR 2078851

Received November 19, 2004; revised June 1, 2005

Nikolaos Diamantis, School of Mathematical Sciences, University of Nottingham, Nottingham, NG7 2RD, United Kingdom

E-mail: nikolaos.diamantis@ nottingham.ac.uk

Ramesh Sreekantan, School of Mathematics, Tata Institute of Fundamental Research, Dr Homi Bhabha Rd, Bombay 400 005, India

E-mail: ramesh@math.tifr.res.in 